\title{
Safety and efficacy of lifitegrast 5\% ophthalmic solution in contact lens discomfort
}

This article was published in the following Dove Press journal: Clinical Ophthalmology

Agustin L Gonzalez

Eye \& Vision, Richardson, TX 75082, USA
Correspondence: Agustin L Gonzalez Eye \& Vision, 3417 Spectrum Boulevard, Richardson, TX 75082, USA

Tel + I 214392 |77|

Email gonzalez.agu@gmail.com
Purpose: The aim of this study was to evaluate the safety and efficacy of lifitegrast $5 \%$ ophthalmic solution in improving contact lens discomfort (CLD) in patients wearing a lowmodulus, nonionic, monthly replacement silicone hydrogel contact lenses (SHCLs).

Patients and methods: A single center, prospective, open-label study was undertaken on patients wearing monthly replacement, low modulus silicone hydrogel contact lenses. Bestcorrected visual acuity (BVA), lissamine staining (LS) and eight item contact lens dry eye questionnaire (CLDEQ-8) scores were assessed at baseline and after 8-week therapy of twicea-day lifitegrast 5\% ophthalmic solution use. Pre- and Posttreatment data were analyzed for statistical significance.

Results: Twenty-one subjects ( 15 female and six male) with the mean age of $31.7( \pm 5.29)$ years completed the study. No reduction or statistically significant change in monocular BVA or LS scores was observed. Data showed a statistically significant $(P<0.05)$ reduction in total CLDEQ-8 score, questions $1 \mathrm{~A}, 1 \mathrm{~B}, 2 \mathrm{~A}$ and $2 \mathrm{~B}$ scores and nonstatistically significant reduction in questions $3 \mathrm{~A}, 3 \mathrm{~B}, 4$ and 5 scores. Fifteen $(\mathrm{n}=15,71.4 \%)$ study subjects found the therapy positive for their contact lens wear.

Conclusion: Data suggest the use of topical lifitegrast 5\% ophthalmic solution twice a day may be a safe and effective therapeutic intervention for managing patients with CLD in SHCL users; however further research is indicated.

Keywords: lifitegrast, contact lens discomfort, dry eye, contact lens discontinuation

\section{Introduction}

Symptoms of dryness remain a problem for contact lens wearers, and the management of contact lens discomfort (CLD) remains a challenge in the contact lens industry and for the practitioners involved in clinical contact lens practice alike. ${ }^{1}$ A report by the International Workshop on CLD, organized by the Tear Film and Ocular Surface Society (TFOS) published in Investigative Ophthalmology and Visual Science, volume 54, No 11, October 2013, defined and extensively discussed the multiple aspects of this topic. ${ }^{2-10}$ The paper noted the role of inflammation as one of the many probable causes of CLD. ${ }^{10}$

Research studies on silicone hydrogel contact lens (SHCL) design, polymer technology, and SHCL products aim to develop a combination of properties that minimize the impact on eye health and maximize wearing comfort. Modifications in design, polymers, cleaning disinfecting products, and coatings aim to extend lens wear and comfort to improve user contact lens wearing experience (CLWE). ${ }^{4,6,8}$ The product design adjusts and accounts for the interaction of the lens and ocular surface, balancing both mechanical and physiological effects on the eye. ${ }^{4,6,8}$ With all this knowledge and developments in contact lens technology, the symptom of dryness-related discomfort 
has still been shown to be the predominant reason for discontinuation of contact lens wear and frustration in both patients and clinicians. ${ }^{1}$

Integrins mediate cell-to-cell interactions and play a role in the inflammatory cascade of dry eye disease. Intercellular adhesion molecule 1 (ICAM-1) is expressed in several cells, including endothelial, epithelial, and antigen-presenting cells. ${ }^{11-14}$ ICAM-1 is overexpressed in patients with dry eye with the binding between lymphocyte function-associated antigen 1 (LFA-1) and ICAM-1 described as an "immunological synapse" and critical initial step in T-cell activation and mediation of inflammation. ${ }^{11-14}$

Lifitegrast 5\% ophthalmic solution (Xiidra ${ }^{\circledR}$; Shire, Lexington, MA, USA) is a topically administered ophthalmic eye drop approved in the USA by the Food Drug Administration (FDA) as a first-in-class drug called LFA-1 antagonist and new treatment option for signs and symptoms of dry eye disease. Lifitegrast 5\% ophthalmic solution is a small molecule antagonist of the LFA-1 integrin that inhibits the binding of LFA-1 to ICAM-1. Lifitegrast 5\% ophthalmic solution is applied twice a day topically, and clinical trials have proven it as safe and effective in the treatment of signs and symptoms of dry eye disease. ${ }^{15-22}$ A recently published study reported lifitegrast 5\% ophthalmic solution as being effective in reducing symptoms of eye dryness. ${ }^{23}$

Validated questionnaires help in quantifying subjective complaints and can provide a useful tool for monitoring an individual's perception of changes with therapeutic interventions. The eight-item Contact Lens Dry Eye Questionnaire (CLDEQ-8) is a validated eight-item scaled questionnaire developed to reflect the overall opinion or status of change of soft contact lenses (SCLs) including SCHL, and it is useful in evaluating CLD. ${ }^{24}$ It has been designed by grouped items evaluating late-day dryness and discomfort, blurriness of vision, and desire to close the eyes and remove SCLs to relieve discomfort.

The CLDEQ-8 has been recognized as a useful tool to reflect the status and change in the overall opinion after refitting SCLs to compare two types of SCL wearing experiences. ${ }^{7}$ CLDEQ-8 scoring is highly correlated with contact lens wearers' baseline opinion of the CLWE and dryness. ${ }^{25}$

There is an acknowledgment that inflammation does play a role in CLD. ${ }^{26,27}$ Understanding it is the sensation of eye dryness-related discomfort that often perplexes clinicians in managing CLD and causes discontinuation of habitual contact lens wear in patients, in the present study, the author wanted to evaluate the use of lifitegrast $5 \%$ ophthalmic solution as a therapeutic intervention for CLD. The study was designed to evaluated safety of lifitegrast $5 \%$ ophthalmic solution using best-corrected visual acuity (BVA) and lissamine staining (LS) and efficacy using CLDEQ-8 scores in patients wearing a low modulus, non-ionic, monthly replacement SHCL over an eight-week test period.

\section{Patients and methods}

The study was undertaken in compliance with Good Clinical Practice guidelines and the tenets of the Declaration of Helsinki after the study protocol, informed consent form including the acknowledgment of off-label use of lifitegrast $5 \%$ ophthalmic solution, investigator qualifications, clinical site, and recruiting materials were reviewed and approved by EV Clinical Trials Institutional Review Board committee. All the visits were conducted by the author and a technician in a clinical setting. The study protocol included the collection of authorization and informed consent to use health research study information. The study was designed as a single-center, open-label, prospective trial on the effect of lifitegrast $5 \%$ ophthalmic solution on CLD on patients wearing a lowmodulus nonionic SHCL.

Subjects were recruited through in-office recruitment. Inclusion criteria were as follows: a minimum of 20 years of age, a minimum of previous 10-month history of self-assessed comfortable wear of Bausch \& Lomb Ultra (BLUltra) with Moisture Seal technology ${ }^{\circledR}$ (Bausch \& Lomb Incorporated, Bridgewater, NJ, USA), a nonionic silicone hydrogel castmolded samfilcon A lens with $46 \%$ water content, Dk of 114 (Dk/t 163), and a modulus of 70. All subjects stated that they were not using any additional medications or therapy to obtain improved contact lens comfort and also that they were wearing contact lens for a minimum of 8 hours a day.

Exclusion criteria included self-reported symptomatic intolerance of contact lenses, a history of an active anterior segment, eyelids, eyelashes, corneal or conjunctival pathology, ongoing ocular infection, inflammation, or any other ocular problems that would require management with topical ophthalmic medications. Demographic data and ocular and general medical history were all recorded as part of the initial encounter, and a comprehensive eye health assessment was performed with any active medications or over-the-counter (OTC) products; supplements were recorded. Potential childbearing women were required to state they were not pregnant or intending to get pregnant during the study period.

Internal and external eye health assessments were performed via slit-lamp biomicroscopy. Contact lens refitting protocol included monocular refraction for BVA, cornea, and conjunctival assessment using LS measured using the Oxford Schema scoring method and in vivo contact lens centration, fit, and movement evaluation to assure adequate contact lens fit. 
All the subjects enrolled continued using the BLUltra, an FDA-approved, daily-wear, 1-month disposable cast-molded SHCL. At initial examination, subjects were considered at day 1 of a 4-week replacement schedule for contact lenses and given a 60-day supply of contact lenses consisting of two pairs (four lenses, the initial pair and the second pair) of BLUltra contact lenses with instructions to discard the initial pair on day 30 of continuous daily wear and initiate the second pair on day 31. BLUltra contact lens wearing instruction included the use of contact lenses encouraged during a minimum of 8 hours a day with no overnight wear and following cleaning instructions as per manufacturer's recommendations with overnight soaking and disinfection of contact lenses using Biotrue $^{\circledR}$ (Bausch \& Lomb Incorporated) cleaning/disinfecting solution, supplied for study purposes. Safety end points were defined as no reduction in BVA, no increase in LS scores at study completion, and no discontinuation of therapy.

The first dose of study medication was self-administered in office before contact lens insertion, and instillation was supervised at the clinic site with instillation and dosing instructions provided. As per package insert, subjects were instructed to wait a minimum of 15 minutes after the instillation of lifitegrast before the insertion of the contact lenses. Lifitegrast use instructions were based on the labeled FDAapproved use of one drop twice a day applied topically to each eye. A review of most frequently reported ocular adverse events (AEs) was discussed including intermittent instillation site signs and symptoms such as irritation, discomfort, blurred vision, and dysgeusia.

The subjects were discouraged but not prohibited from using any current OTC drops during the study period. They were instructed not to use any other previously prescribed topical ophthalmic medications nor prescribed concomitant therapy during the study period. Topical medication not allowed to be used included topical corticosteroids, nonsteroidal anti-inflammatory agents, and topical antihistamine either prescribed or OTC. Use of contact lens indicated that OTC lubricating topical eye drops were discouraged.

The subjects were queried by the technician via phone for major AEs, defined as requiring discontinuation of lifitegrast $5 \%$ ophthalmic solution, relating to therapy or contact lenses after 7 days of initiation in the study. No major AEs were reported or noted at this time. After 8 weeks, the subjects were scheduled and evaluated for a second and final study visit at which time subjects were queried for AEs relating to therapy and contact lens use. No concomitant medication usage was recorded for any subject. No major (defined as needing to discontinue therapy) AEs were recorded. The study subjects underwent and were assessed for monocular
BCVA without contact lenses, biomicroscope, and external eye examination and ocular surface evaluation by LS staining (Oxford Schema grading).

The subjects were instructed to complete the second final CLDEQ-8 questionnaire and asked verbally to reply to the statement read to them: "do you believe the therapy was a positive, neutral, or negative in your contact lens wearing experience," and the subjects' verbal responses were recorded. At this time, the subjects were informed that the study was concluded and were instructed to discard their current pair of lenses and resume regular contact lens wear routine. A new pair of BLUltra contact lenses with Biotrue cleaning solutions was dispensed at this moment. Post-study data were analyzed for statistical significance.

\section{Results}

Statistical analysis was performed using SPSS 17.0 for Windows (SPSS Inc, Chicago, Illinois, USA) with descriptive data presented as mean ( \pm standard deviation) unless noted otherwise. A total of twenty-one (21) subjects were enrolled and completed this study: 15 (71.4\%) women, six (28.5\%) men. The study group had a mean age of $31.76( \pm 5.29)$ years, ranging from 21 to 41 years. Women's average age was $32.33( \pm 5.37)$ years, and men's average age was 30.33 $( \pm 4.78)$ years. The mean time interval between the baseline and follow-up appointment was 64.05 days $( \pm 4.69)$ with a range of 55 to 74 days (Table 1).

\section{Safety}

There were no statistically significant $(P>0.05)$ changes in BVA or LS Oxford Schema scores noted after 8 weeks. Minor reported AE's after eight weeks included two subjects ( $\mathrm{n}=2$, 9.5\%) reported mild stinging at site instillation during the study period, and one $(\mathrm{n}=1,0.5 \%)$ reported an occasional, transient mild taste disruption. No serious AEs requiring discontinuation of treatment were reported or noted in any subject.

\section{Efficacy}

At baseline visit, symptom severity data collected by the CLDEQ-8 scores ranged from 10 to 15 with an average of

Table I Age and gender distribution at the second visit, time period in days

\begin{tabular}{|l|l|l|l|}
\hline Gender & $\mathbf{n}$ & Average age, years & Average days \\
\hline Male & 6 & $30.33 \pm 4.78$ & $64.27 \pm 5.22$ \\
\hline Female & 15 & $32.33 \pm 5.37$ & $63.50 \pm 2.87$ \\
\hline Total & 21 & $31.76 \pm 5.29$ & $64.05 \pm 4.69$ \\
\hline
\end{tabular}

Note: Data are presented as mean \pm standard deviation. 
Table 2 Total and item average baseline and final CLDEQ-8 scores and $P$ value

\begin{tabular}{|l|l|l|l|}
\hline $\begin{array}{l}\text { CLDEQ8 } \\
\text { items }\end{array}$ & $\begin{array}{l}\text { Initial } \\
\text { average }\end{array}$ & $\begin{array}{l}\text { Final } \\
\text { average }\end{array}$ & $P$-value \\
\hline $\mathrm{Ia}$ & $2.19 \pm 0.39$ & $1.86 \pm 0.35$ & 0.002 \\
\hline $\mathrm{Ib}$ & $1.62 \pm 0.58$ & $1.48 \pm 0.50$ & $0.04 \mathrm{I}$ \\
\hline $2 \mathrm{a}$ & $2.38 \pm 0.65$ & $1.86 \pm 0.47$ & 0.002 \\
\hline $2 \mathrm{~b}$ & $1.67 \pm 0.56$ & $1.38 \pm 0.49$ & 0.015 \\
\hline $3 \mathrm{a}$ & $1.48 \pm 0.50$ & $1.38 \pm 0.49$ & $0.08 \mathrm{I}$ \\
\hline $3 \mathrm{~b}$ & $1.29 \pm 0.45$ & $1.19 \pm 0.39$ & $0.08 \mathrm{I}$ \\
\hline 4 & $1.57 \pm 0.66$ & $1.52 \pm 0.50$ & 0.358 \\
\hline 5 & $1.10 \pm 0.6 \mathrm{I}$ & $0.95 \pm 0.65$ & 0.133 \\
\hline Total & $13.29 \pm 1.35$ & $11.62 \pm 0.99$ & 0.000 \\
\hline
\end{tabular}

Notes: Data presented as mean \pm SD baseline to final and $P$ value from to baseline. CLDEQ-8 = eight item Contact Lens Dry Eye Questionnaire.

Abbreviation: CLDEQ-8, eight-item Contact Lens Dry Eye Questionnaire.

13.29 ( \pm 1.35$)$. At study completion, the symptom severity data collected by the CLDEQ-8 scores ranged from 9 to 13 with an average of $11.62( \pm 0.99)$. Table 2 summarizes the average initial and final CLDEQ-8 scores.

There was an average 1.68-point reduction change in the total response between the pre- and post-therapy administration of the CLDEQ-8 and an average item reduction of $0.20( \pm 0.15)$. The initial and final CLDEQ-8 scores with changes are shown in Figure 1.

Item 1a asked about the frequency of eye discomfort with habitual contact lens wear during a typical day in the past 2-week period showed an average reduction of $0.33( \pm 0.35)$, while $1 \mathrm{~b}$ asked about the intensity of this discomfort at the end of the wearing time showed an average reduction of $0.14( \pm 0.50)$. Item $2 \mathrm{a}$ asked about the frequency of eye dryness with habitual contact lens wear during a typical day in the past 2-week period showed the biggest item average reduction of $0.52( \pm 0.47)$, while $2 \mathrm{~b}$ asked about the intensity of this dryness at the end of the wearing time showed an average reduction of $0.29( \pm 0.49)$.

Item $3 \mathrm{a}$ asked about the frequency of changeable blurry vision with habitual contact lens wear during a typical day in the past 2-week period and $3 \mathrm{~b}$ asked about how notable was the changeable blurry vision at the end of the wearing time showed similar average reductions of $0.10( \pm 0.49)$ and $0.10( \pm 0.39)$, respectively. When asked about the frequency of wanting to close the eyes to improve the sensation of bothersome with habitual contact lens wear during the past 2-week period, item 4 achieved the lowest average change with $0.05( \pm 0.50)$. When asked about the frequency of wanting to remove the contact lenses to improve eye discomfort with habitual contact lens wear during the past 2-week period, item 4 achieved an average change of $0.14( \pm 0.65)$. Six subjects $(n=6,28.5 \%)$ found the therapy as a neutral experience in lens wear, while $15(\mathrm{n}=15,71.4 \%)$ perceived the therapy as positive, and no subject found the intervention negative.

Pre- and post-therapy total and single-item CLDEQ-8 scores were compared using paired $t$-test. Normality of scores was assumed with a null hypothesis of no difference between the pre- and posttreatment values. The $P$-values presented are one-sided and evaluated using a 0.05 confidence level. The total and separate CLDEQ-8 item scores were analyzed.

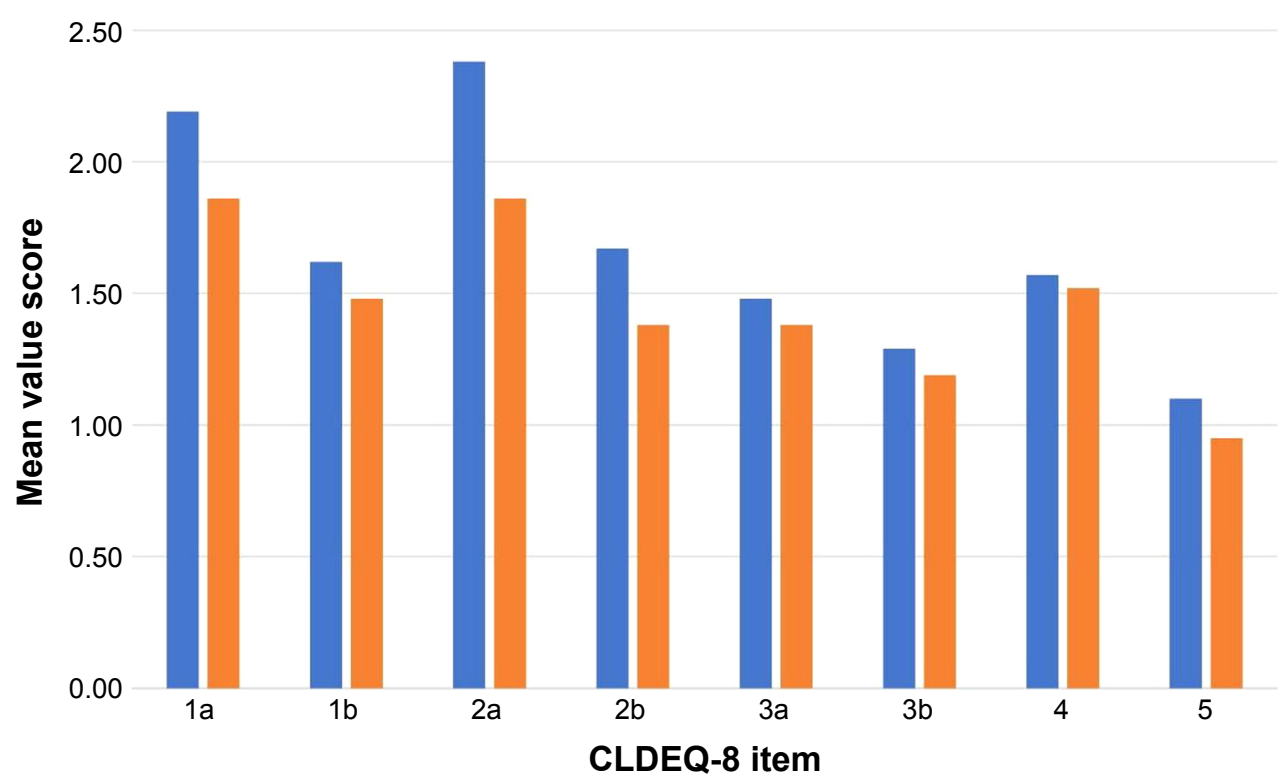

Figure I Baseline and final CLDEQ-8 item score.

Notes: Comparison of the eight item Contact Lens Dry Eye Questionnaire (CLDEQ-8) Baseline (initial) and final item scores after using lifitegrast $5 \%$ ophthalmic solution treatment (data are mean \pm SD). A lower score means reduced symptoms. Improvements in items la, Ib, 2a, 2b were statistically significant $(P<0.05)$. Abbreviation: CLDEQ-8, eight-item Contact Lens Dry Eye Questionnaire. 
The total overall pre- and post-scores indicate that the mean total CLDEQ-8 scores are statistically significant and seen favoring post-therapy results ( $P \leq 0.0001)$. A statistically significant reduction was observed in items 1 a $(P=0.0024)$, $1 \mathrm{~b}(P=0.0414), 2 \mathrm{a}(P=0.0022)$, and $2 \mathrm{~b}(P=0.0150)$. Nonstatistically significant $(P>0.05)$ reduction was noted in items 3a $(P=0.0811), 3$ b $(P=0.0811), 4(P=0.3576)$, and 5 $(P=0.1335$; Table 2$)$.

Comparing the male to female cohort, both total mean pretreatment $(P=0.063)$ and post-treatment $(P=0.557)$ scores did not differ significantly from one another. Both populations mean initial score is statistically significantly from the mean final scores for both females $(P=0.0001)$ and males $(P=0.0026)$.

Analyzing verbal response type (neutral and positive) both the neutral group $(P=0.0172)$ the positive group $(P<0.0001)$ showed statistically significant differences in total CLDEQ-8 scores from initial to final. When looking at days in therapy, for every one-unit increase in days there was a 0.124 -point decrease in CLDEQ-8 scores suggesting there may be an association between duration of treatment and post-therapy score as the duration of therapy is extended $(P=0.0055)$.

\section{Discussion}

Our understanding and knowledge of CLD have greatly expanded in the past decade..$^{2-9}$ Even with this knowledge and advances in contact lens material, design, and wearing modalities all aimed at giving both patients' and clinicians' options, there is still CLD that drives patients to discontinue contact lens wear. ${ }^{1}$ The approval of lifitegrast $5 \%$ ophthalmic solution as a new therapeutic intervention has provided clinicians a safe, new treatment choice for managing the signs and symptoms of eye dryness. ${ }^{15-23}$

Available interventions for managing CLD include contact lens-lubricating eye drops, rewetting, and supplementation by artificial tear formulations considered by many practitioners as an initial step for SHCL wearers who experience CLD. Current consensus supports the use of tear supplementation, contact lens lubrication, and rewetting drops for the relief of dryness; although beneficial in managing symptoms, these remedies mostly provide temporary relief..$^{8,9}$

Many variables are accounted for in the clinical decisionmaking process in managing patients' desires to use SCL and their complications. The origin of CLD is very complex with mechanical, physiological, biological, and immunological factors among others contributing to the symptoms of CLD considered by clinicians. ${ }^{2}$ Contact lens-specific factors such as contact lens material, replacement schedules, and contact lens care solutions must be weighed with ocular factors such as tear film composition, stability osmolarity, lid interaction, and all placed in the context of medical, psychological, and demographic variables. ${ }^{2}$ The understanding of the ocular surface and cellular and biochemical inflammatory cycles has evolved, and it is becoming evident that clinicians are acknowledging and accepting that the CLWE, even when uncomplicated, is naturally inflammatory. ${ }^{26,27}$

Contemporary clinical opinion is starting to revisit the CLD and CLWE outside of the binary mechanical/disease entity boundaries and possibly starting to recognize an associated subclinical inflammatory-mediated process. ${ }^{9}$ As has been suggested, this subjective comfort/discomfort contact lens experience could be driven by the upregulation of the immune system maintaining the eye in an immunological and pre-inflammatory state of alert in the presence of a contact lens interacting with the ocular surface. Efron $^{27}$ has been one of the authors who had very eloquently argued this chronic, low-level, non-damaging upregulation of the immune system as subclinical inflammation in contact lens wear.

Consideration must then be given to the emergent contemporary way of thinking about the role of chronic, low-grade, subclinical inflammatory status of the ocular surface during contact lens wear and the role it plays in the SHCL wearing experience and discontinuation. Understanding of the inflammatory process and pharmacological strategies that modulate inflammation seems to have the potential to help clinicians in positively influencing the patients' SHCL wearing experience.

The author chose the CLDEQ-8 as a tool to measure a symptom-based condition from the contact lens users' perspective and a well-recognized tool in assessing CLD. ${ }^{7,24,25}$ In this study, the CLDEQ-8 test proved to be a very valuable tool in quantifying symptoms by individual SCL wearers in capturing the patient-reported opinion of their CLWE with the treatment of lifitegrast $5 \%$ ophthalmic solution.

The author recognizes multiple limitations of this openlabel, prospective study, primarily a lack of control group with randomization, little information on performance on other modalities or types of contact lenses, no recorded users wearing schedules or daily activity affecting CLWE, comorbidities, lack of information on specific ocular or systemic etiology underlying any dry eye disease for this study population, and limited safety metrics. Even acknowledging the study limitations, the results are encouraging.

To the author's knowledge, this is the first reported clinical study examining the effect of lifitegrast $5 \%$ ophthalmic solution as a management option for CLD. Based on the FDAapproved indication for signs and symptoms of dryness, the author decided to evaluate lifitegrast $5 \%$ ophthalmic solution 
as a management option for CLD. The findings suggest that the possibility of twice-a-day use of topical lifitegrast $5 \%$ ophthalmic solution might be an effective intervention to improve CLD in SHCL users.

\section{Conclusion}

In this study population of SHCL wearers, CLDEQ-8 items $1 \mathrm{a}$ and $1 \mathrm{~b}$, items $2 \mathrm{a}$ and $2 \mathrm{~b}$, and total CLDEQ- 8 scores showed a statistically significant improvement with the use of lifitegrast $5 \%$ ophthalmic solution. The results of this study indicate lifitegrast 5\% ophthalmic solution may be a safe and effective therapeutic intervention in managing patients with CLD. The role of lifitegrast $5 \%$ ophthalmic solution in CLD should be considered for further studies.

\section{Disclosure}

Dr Agustin L Gonzalez is a consultant and speaker for Shire Ophthalmics and Bausch+Lomb Vision Care. The author reports no other conflict of interest in this work.

\section{References}

1. Nichols JJ, Willcox MD, Bron AJ, et al. The TFOS International Workshop on Contact Lens Discomfort: executive summary. Invest Ophthalmol Vis Sci. 2013;54(11):TFOS7-TFOS13.

2. Nichols JJ, Jones L, Nelson JD, et al. The TFOS International Workshop on Contact Lens Discomfort: introduction. Invest Ophthalmol Vis Sci. 2013;54(11):TFOS1-TFOS6.

3. Nichols KK, Redfern RL, Jacob JT, et al. The TFOS International Workshop on Contact Lens Discomfort: report of the definition and classification subcommittee. Invest Ophthalmol Vis Sci. 2013;54(11): TFOS14-TFOS19.

4. Craig JP, Willcox MD, Argüeso P, et al. The TFOS International Workshop on Contact Lens Discomfort: report of the contact lens interactions with the tear film subcommittee. Invest Ophthalmol Vis Sci. 2013;54(11):TFOS123-TFOS156.

5. Dumbleton K, Caffery B, Dogru M, et al. The TFOS International Workshop on Contact Lens Discomfort: report of the subcommittee on epidemiology. Invest Ophthalmol Vis Sci. 2013;54(11):TFOS20-TFOS36.

6. Efron N, Jones L, Bron AJ, et al. The TFOS International Workshop on Contact Lens Discomfort: report of the contact lens interactions with the ocular surface and adnexa subcommittee. Invest Ophthalmol Vis Sci. 2013;54(11):TFOS98-TFOS122.

7. Foulks G, Chalmers R, Keir N, et al. The TFOS International Workshop on Contact Lens Discomfort: report of the subcommittee on clinical trial design and outcomes. Invest Ophthalmol Vis Sci. 2013;54(11): TFOS157-TFOS183.

8. Jones L, Brennan NA, González-Méijome J, et al. The TFOS International Workshop on Contact Lens Discomfort: report of the contact lens materials, design, and care subcommittee. Invest Ophthalmol Vis Sci. 2013;54(11):TFOS37-TFOS70.
9. Papas EB, Ciolino JB, Jacobs D, et al. The TFOS International Workshop on Contact Lens Discomfort: report of the management and therapy subcommittee. Invest Ophthalmol Vis Sci. 2013;54(11): TFOS183-TFOS203.

10. Stapleton F, Marfurt C, Golebiowski B, et al. The TFOS International Workshop on Contact Lens Discomfort: report of the subcommittee on neurobiology. Invest Ophthalmol Vis Sci. 2013;54(11): TFOS71-TFOS97.

11. Sun Y, Zhang R, Gadek TR, O'Neill CA, Pearlman E. Corneal inflammation is inhibited by the LFA-1 antagonist, lifitegrast (SAR 1118). J Ocul Pharmacol Ther. 2013;29(4):395-402.

12. Perez VL, Pflugfelder SC, Zhang S, Shojaei A, Haque R, Lifitegrast HR. Lifitegrast, a novel integrin antagonist for treatment of dry eye disease. Ocul Surf. 2016;14(2):207-215.

13. Semba CP, Gadek TR. Development of lifitegrast: a novel T-cell inhibitor for the treatment of dry eye disease. Clin Ophthalmol. 2016;10: 1083-1094.

14. Pflugfelder SC, Stern M, Zhang S, Shojaei A. LFA-1/ICAM-1 Interaction as a therapeutic target in dry eye disease. J Ocul Pharmacol Ther. 2017;33(1):5-12.

15. Donnenfeld ED, Karpecki PM, Majmudar PA, et al. Safety of Lifitegrast Ophthalmic Solution 5.0\% in Patients With Dry Eye Disease: A 1-Year, Multicenter, Randomized, Placebo-Controlled Study. Cornea. 2016;35(6):741-748.

16. Holland EJ, Luchs J, Karpecki PM, et al. Lifitegrast for the Treatment of Dry Eye Disease: Results of a Phase III, Randomized, Double-Masked, PlaceboControlled Trial (OPUS-3). Ophthalmology. 2017;124(1):53-60.

17. Semba CP, Swearingen D, Smith VL, et al. Safety and pharmacokinetics of a novel lymphocyte function-associated antigen-1 antagonist ophthalmic solution (SAR 1118) in healthy adults. J Ocul Pharmacol Ther. 2011;27(1):99-104.

18. Holland EJ, Whitley WO, Sall K, et al. Lifitegrast clinical efficacy for treatment of signs and symptoms of dry eye disease across three randomized controlled trials. Curr Med Res Opin. 2016:1-7.

19. Nichols KK, Donnenfeld ED, Karpecki PM, et al. Safety and tolerability of lifitegrast ophthalmic solution 5.0\%: Pooled analysis of five randomized controlled trials in dry eye disease. Eur J Ophthalmol. 2018;1120672118791936.

20. Nichols KK, Holland E, Toyos MM, et al. Ocular comfort assessment of lifitegrast ophthalmic solution 5.0\% in OPUS-3, a Phase III randomized controlled trial. Clin Ophthalmol. 2018;12:263-270.

21. Paton DM. Lifitegrast: First LFA-1/ICAM-1 antagonist for treatment of dry eye disease. Drugs Today. 2016;52(9):485-493.

22. Godin MR, Gupta PK. Lifitegrast ophthalmic solution in the treatment of signs and symptoms of dry eye disease: design, development, and place in therapy. Clin Ophthalmol. 2017;11:951-957.

23. De Paz CJ, Gonzalez AL, Ngo C. SIDRE: Symptomatic improvement of dry eye study. Clin Optom. 2017;9:49-54.

24. Chalmers RL, Begley CG, Moody K, Hickson-Curran SB. Contact Lens Dry Eye Questionnaire-8 (CLDEQ-8) and opinion of contact lens performance. Optom Vis Sci. 2012;89(10):1435-1442.

25. Chalmers RL, Keay L, Hickson-Curran SB, Gleason WJ. Cutoff score and responsiveness of the 8-item Contact Lens Dry Eye Questionnaire (CLDEQ-8) in a Large daily disposable contact lens registry. Cont Lens Anterior Eye. 2016;39(5):342-352.

26. Efron N. Rethinking contact lens discomfort. Clin Exp Optom. 2018; 101(1):1-3.

27. Efron N. Contact lens wear is intrinsically inflammatory. Clin Exp Optom. 2017;100(1):3-19. 
Clinical Ophthalmology

\section{Publish your work in this journal}

Clinical Ophthalmology is an international, peer-reviewed journal covering all subspecialties within ophthalmology. Key topics include: Optometry; Visual science; Pharmacology and drug therapy in eye diseases; Basic Sciences; Primary and Secondary eye care; Patien Safety and Quality of Care Improvements. This journal is indexed on

Submit your manuscript here: http://www.dovepress.com/clinical-ophthalmology-journal

PubMed Central and CAS, and is the official journal of The Society of Clinical Ophthalmology (SCO). The manuscript management system is completely online and includes a very quick and fair peer-review system, which is all easy to use. Visit http://www.dovepress.com/ testimonials.php to read real quotes from published authors. 